\title{
ACHONDROGENESIS TYPE 2 IN A NEWBORN WITH A NOVEL MUTATION ON THE COL2A1 GENE
}

\author{
Dogan $\mathrm{P}^{1, *}$, Varal IG ${ }^{1}$, Gorukmez $\mathrm{O}^{2}, \mathrm{Akkurt}_{\mathrm{MO}^{3}}$, Akdag $\mathrm{A}^{1}$ \\ *Corresponding Author: Pelin Dogan, M.D., University of Health Sciences, Bursa Yuksek Ihtisas \\ Teaching Hospital, Department of Pediatrics, Division of Neonatology, Y1ldırım, Bursa, Turkey. \\ Tel.: +90-505-316-4268. Fax: +90-224-294-4000. E-mail: pelin_akbas@yahoo.com
}

\begin{abstract}
Achondrogenesis is a group of rare and fatal disorders occurring in approximately one in every 40,000-60,000 newborns. Achondrogenesis is classified in three groups, as Achondrogenesis type 1A (Houston-Harris type or ACG1A), Achondrogenesis type 1B (Parenti-Fraccaro type or ACG1B) and Achondrogenesis type 2 (Langer-Saldino type or ACG2), depending on clinical and radiological findings. Achondrogenesis Type 2 is a lethal skeletal dysplasia that is typically characterized by short arms and legs, a small chest with short ribs, lung hypoplasia, a prominent forehead, a small chin, and an enlarged abdomen that may accompanied by polydramnios and hydrops. This study contributes to the literature by presenting a patient who was admitted to the Level III Neonatal Intensive Care Unit (NICU), Bursa, Turkey), with extremely short extremities, a small chest, abdominal distention and respiratory distress, who was diagnosed with ACG2. On the COL2A1 gene, genetic analysis with next generation sequencing (NGS), was revealed to have a heterozygous missense variation, c. $2546 \mathrm{G}>\mathrm{A}$, p.Gly849Asp mutation, which is a different genetic variant that has not been previously described in the literature.
\end{abstract}

Keywords: Achondrogenesis type 2 (ACG2); COL2A1 mutation; Lethal skeletal dysplasia.

\footnotetext{
${ }^{1}$ Department of Pediatrics, Division of Neonatology, University of Health Sciences, Bursa Yuksek Ihtisas Teaching Hospital, Bursa, Turkey

${ }^{2}$ Department of Genetics, University of Health Sciences, Bursa Yuksek Ihtisas Teaching Hospital, Bursa, Turkey

${ }^{3}$ Department of Obstetrics and Gynecology, Division of Perinatology, University of Health Sciences, Bursa Yuksek Ihtisas Teaching Hospital, Bursa, Turkey
}

\section{INTRODUCTION}

Achondrogenesis is a group of rare and fatal disorders occurring in approximately one in every 40,000-60,000 newborns [1]. It is clinically characterized by short-limbed dwarfism and the affected patients are often lost in utero or shortly after birth. Achondrogenesis is classified as Achon-rogenesis type 1A (Houston-Harris type or ACG1A), Achondrogenesis type 1B (Parenti-Fraccaro type or ACG1B) and Achondrogenesis type 2 (Langer-Saldino type or ACG2), depending on clinical and radiological findings [2]. Achondrogenesis type 2 is typically characterized by short arms and legs, a small chest with short ribs, lung hypoplasia, a prominent forehead, a small chin, and an enlarged abdomen that may be accompanied by polyhydramnios and hydrops. Typical radiological findings of ACG2 include short long bones, short ribs without fractures, decreased ossification of the pelvis and spine, and normal mineralization in the iliac and calvarial bones [3].

The COL $2 A 1$ gene encodes the cartilage-specific collagen, and mutations in this gene can cause ACG2 and various skeletal dysplasias. Achondrogenesis type 2 is the most severe and lethal form of the disorder [4]. This study contributes to the literature by presenting a case of ACG2 carrying a heterozygous missense variation (c.2546G $>\mathrm{A}$, p.Gly849Asp in $C O L 2 A 1$ ), a new mutation that has not previously been described in the literature.

\section{CASE REPORT}

A 25-year-old pregnant Syrian woman was referred to the perinatology clinic for further screening due to a suspected skeletal anomaly at 27 weeks' gestation. The previous obstetric history was uneventful and the couple was non consanguineous. The first trimester screening was 
negative, while a detailed sonography scan revealed short long bones ( $<1$ st percentile for all long bones, including femur, tibia, fibula, humerus, radius and ulna) with a small thorax and polihydroamnios. The family was provided genetic counseling related to the high suspicion of lethal skeletal dysplasia. After detailed counseling, a cordocentesis was performed. All sonographic findings suggested skeletal dysplasia; a conventional chromosomal analysis confirmed a normal 46,XY karyotype, while an exome sequencing analysis revealed ACG2. After a 10-week follow-up period, a cesarean delivery was carried out at 39 weeks' gestation due to a previous history of cesarean section.

The patient had a 1 and $5 \mathrm{~min}$. APGAR score of 4 and 5 at birth, respectively. The patient was intubated after delivery due to a lack of spontaneous respiration and was admitted to the Level III Neonatal Intensive Care Unit (NICU), Bursa, Turkey. The birth weight was 2780 gr (3-10 percentile), height was $38 \mathrm{~cm}$ (below 3 percentile), and head circumference was $37 \mathrm{~cm}$ (90-97 percentile). A physical examination revealed extremely short extremities, abdominal distention, a small chest, a prominent forehead and a flat nasal bridge (Figure 1). Ophthalmological exami-

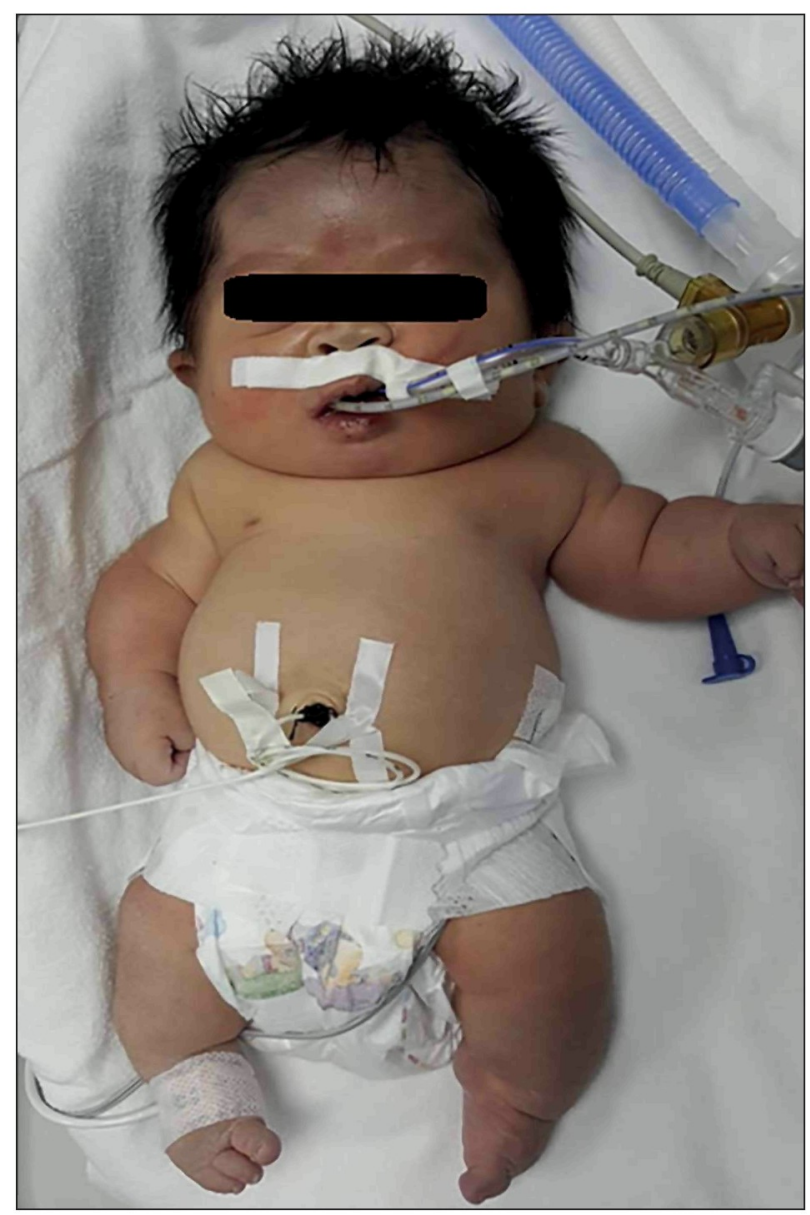

Figure 1. Typical appearance of the patient: short trunk, small thorax, distinctive abdomen and micromelia. nation of the patient was normal. The patient had severe respiratory distress, and follow-up was continued with high frequency oscillation ventilation (HFOV), as conventional mechanical ventilator settings failed to achieve the target saturation level. A peripheral vascular line could not be established due to extensive edema in the body, for which an umbilical catheter was inserted and total parenteral nutrition was initiated.

A cranial and abdominal ultrasound revealed normal findings, while an X-ray examination revealed a short tubular bone structure, metaphyseal widening, short ribs, a small chest and a lack of ossification in the pelvis (Figure 2). A blood sample was obtained for genetic analysis with an antenatal pre diagnosis of ACG2. An echocardiography performed on postnatal day 4 revealed pulmonary hypertension and the patient was administered nitric oxide

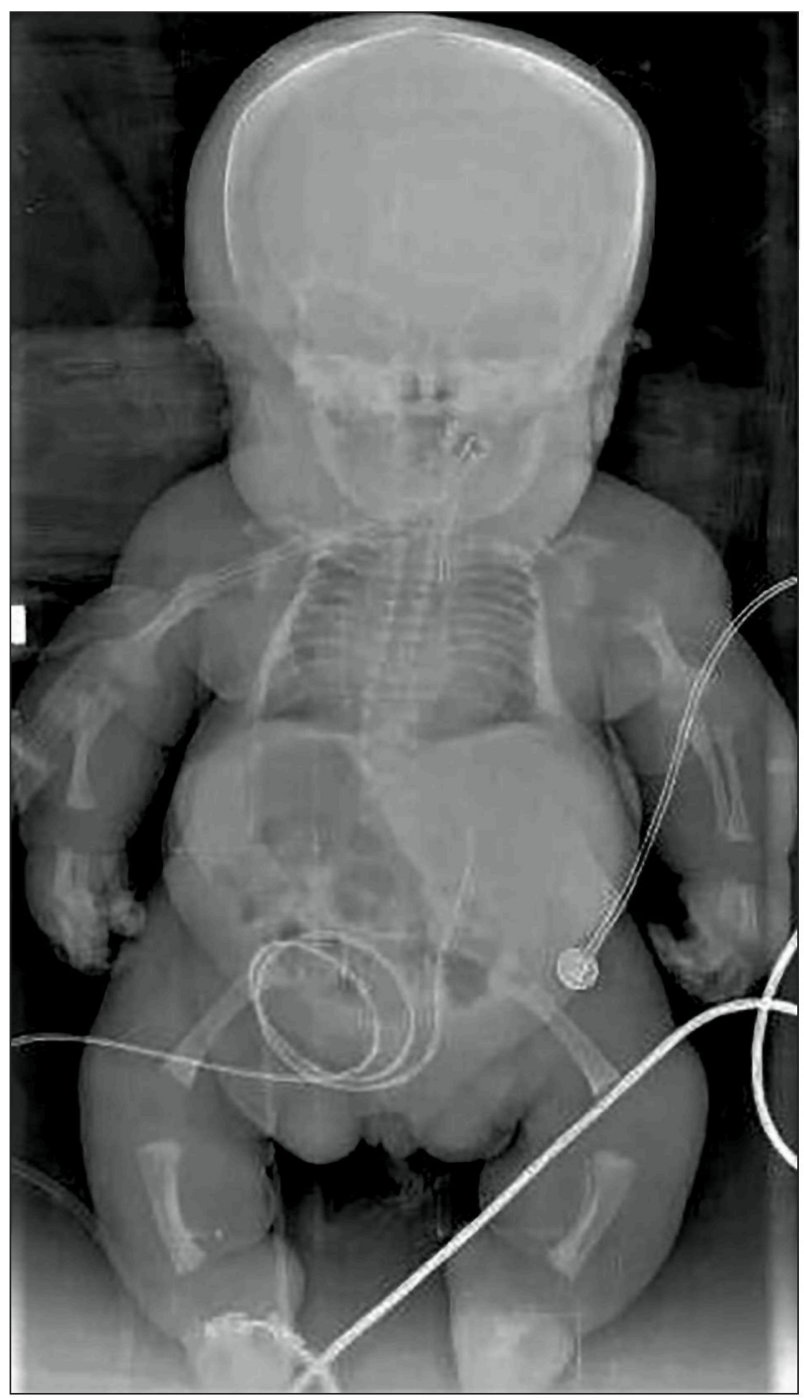

Figure 2. Radiographical findings: short tubular bone with widened metaphyses and non ossified cervical vertebrae, short unfractured ribs, narrow bell-shaped chest, lack of ossification in pelvis and normal ossification of the skull. 
therapy for 2 days. Respiratory distress persisted in the follow-up period and the patient was followed with HFOV due to carbon dioxide retention with conventional ventilator settings. The patient died of respiratory insufficiency on postnatal day 25 .

Genetic Analysis. In the present study, clinical exome sequencing and Sanger sequencing were performed, and genomic DNA was extracted from peripheral venous blood using a QIAamp® DNA Mini Kit (Qiagen, Ankara, Turkey). The Clinical Exome Solution (Sophia Genetics SA, Saint-Sulpice, Switzerland) was used for exome enrichment, with all procedures carried out according to the manufacturer's protocols. This capture-based target enrichment kit covers 4900 genes with known inherited diseases causing mutations.

Paired-end sequencing was performed on a NextSeq 500 system (Illumina, San Diego, CA, USA) with a read length of $150 \times 2$, while the base calling and image analysis were conducted using Real-Time Analysis (integrated to the NextSeq 500 system; Illumina) software. The BCL (base calls) binary is converted into FASTQ utilizing the Illumina package bcl2fastq.

All bioinformatic analyses were performed on a Sophia DDM ${ }^{\mathrm{TM}}$ platform (Sophia Genetics SA), which includes algorithms for alignment, calling single nucleotide polymorphisms (SNPs) and small insertions/deletions (Pepper ${ }^{\mathrm{TM}}$, Sophia Genetics SA patented algorithm) calling copy number variations (Muskat ${ }^{\mathrm{TM}}$, Sophia Genetics SA patented algorithm) and functional annotations (Moka ${ }^{\mathrm{TM}}$, Sophia Genetics SA patented algorithm). The raw reads were aligned to the human reference genome (GRCh37/ hg19). Variant filtering and interpretations were performed on the Sophia DDM ${ }^{\mathrm{TM}}$ platform (Sophia Genetics SA), and an Integrative Genomics Viewer (IGV) was used to visualize the BAM (binary alignment map) files [5]. In families with consanguineous marriages, homozygosity mapping is carried out with HomSI (www.igbam.bilgem. tubitak. gov.tr/softwares/ HomSI) [6]. Next generation sequencing (NGS) showed a heterozygous missense variation, c. $2546 \mathrm{G}>\mathrm{A}$, p.Gly849Asp on the COL2A1 gene that was confirmed by Sanger sequencing (Figure 3).

Genetic Results. This variant (c. $2546 \mathrm{G}>\mathrm{A}$, p.Gly849Asp) on the COL2A1 gene has not been previously reported in the Human Gene Mutation Database (HGMD; http://www.hgmd.cf.ac.uk/ac/index.php) or in population studies (ExAC: Exome Aggregation Consortium and 1000 Genomes Project), while silico analysis programs (VarSome; DANN Score: 0.9969 and ACMG; Likely Pathogenic) have shown that this variation may be the cause of the disease.
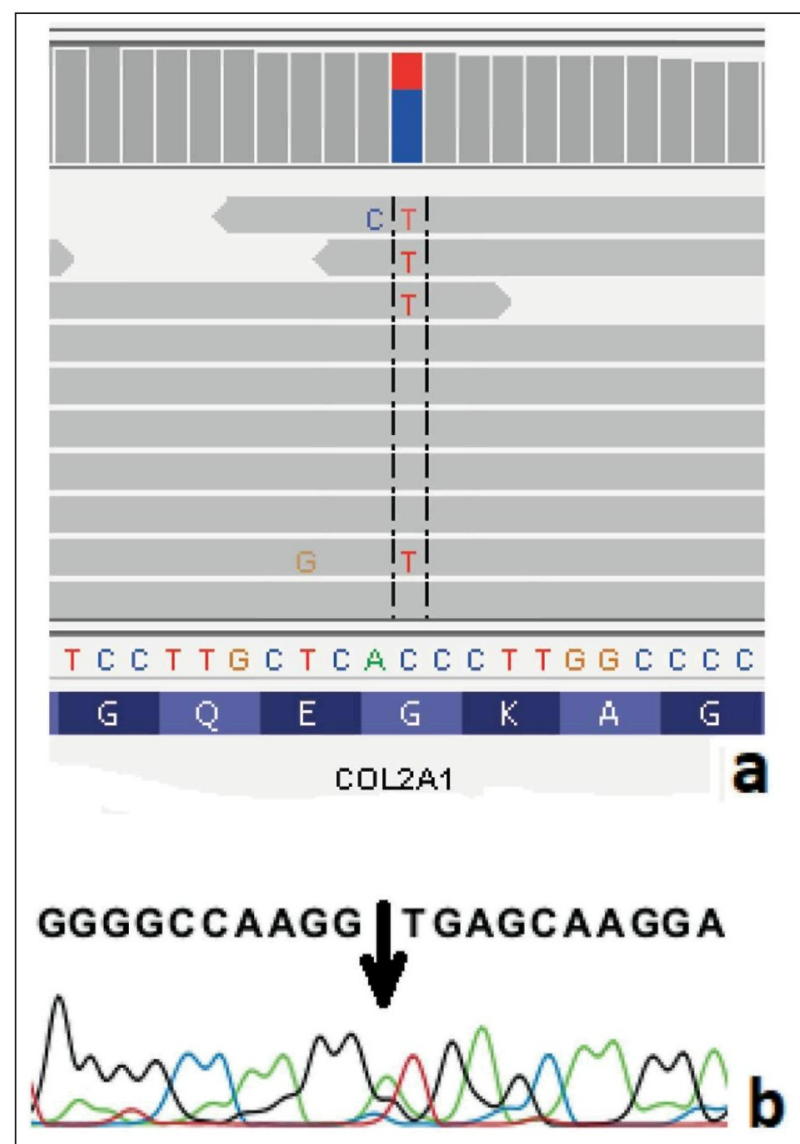

Figure 3. Integrative Genomics Viewer and chromatogram view of the COL2AI gene. (a) Excerpt of exome sequencing data visualized with th Integrative Genomics Viewer. (b) Result of DNA sequencing. A novel heterozygous germline mutation, c. $2456 \mathrm{G}>\mathrm{A}$, p.Gly849Asp on the $C O L 2 A 1$ gene (indicated by a black arrow).

\section{DISCUSSION}

In this report, we present a case with a heterozygous missense variation on the $C O L 2 A 1$ gene, c. $2546 \mathrm{G}>\mathrm{A}$, p.Gly849Asp mutation, being a different genetic variant of ACG2 syndrome that has rarely been reported in the literature. A definitive molecular diagnosis was made in the patient through the application of clinical exome and Sanger sequencing. The fact that the present patient was diagnosed with targeted exome sequencing allowed a shorter diagnostic process than with whole exome sequencing (WES) [7].

The COL2A1 gene located on chromosome 12 encodes polypeptide chains of type 2 collagen. A glycine substitution in the triplehelical domain is the most common type of pathogenic mutation on the COL $2 A 1$ gene [8]. Even though 570 different changes on the COL2AI gene have so far been reported, the genetic variation in 
our case has not been previously reported (c.2546G $>A$, p.Gly849Asp mutation) (HGMD). Genetic variations on the COL2A1 gene are clinically heterogeneous and were associated with 15 different phenotypes (omim.org). In a previous study, it was reported that glycine to asparagine substitutions in COL1A2 resulted in a severe short stature, whereas in another study, it was reported that patients with glycine to asparagine mutations were rather tall $[9,10]$. In the presented case, a glycine to asparagine substitution was observed and a phenotype presented with short structure and shortening of the limbs. In another case, similar to ours, a p.Gly1122Arg mutation was observed as phenotype generalized broadening and shortening of the phalanges were reported [10]. Studies showed that severe ocular phenotype was observed in patients with glycine to aspartic acid and glycine to arginine mutations, but in the presented case, a glycine to asparagine substitution was observed, however, the ophthalmological examination of the patient was normal. The presence of severe mutations affecting glycine is frequent in ACG2 and the frequent mutation was p.(Gly1110Cys) c.3328G $>$ T. Death occurs mostly in utero or during the early neonatal period [11]. Similarly, in the present case, we observed a glycine to asparagine substitution (c.2546G $>$ A, p.Gly849Asp) and the patient died on postnatal day 25. A broad spectrum of disorders occur as a result of mutations on the COL2A1 gene. Milder clinical forms of skeletal dysplasia, such as the Czech dysplasia (OMIM 609192), premature onset arthritis (OMIM 604864), bilateral hip disease and short stature, can occur, but lethal skeletal dysplasias such as ACG2 may also occur [12]. Type 2 collagen is present in the hyaline cartilage and vitreous humor, leading to spinal, epiphyseal and ocular anomalies in COL $2 A 1$ gene mutations.

Dysostoses are constitutional disorders of the bone that take the form of malformations of single or multiple bones in combination. Based on genetic mutations and variations in blastogenesis, they affect normal bone or cartilage development and cause bone defects and skeletal dysplasias [13]. Achondrogenesis comes in three forms, being ACG1A (Houston-Harris type), ACG1B (ParentiFraccaro type) and ACG2 (Langer-Saldino), with differential diagnoses made based on clinical, radiological and molecular findings. The various forms of ACG have identical phenotypic characteristics, such as hydrops fetalis, short neck and trunk, small chest (bell-shaped), prominent abdomen and severe micromyelia, which are typically observed in the all three groups, while there are radiological and molecular differences between the three [13]. The skull is not ossified in ACG1A, whereas there are mildly low ossified areas in ACG1B. Skull ossification is normal in ACG2, alongside decreased ossification in the pelvis and spine. Rib fractures are common in ACG1A, whereas short ribs are often without fractures in ACG2 [2,4,13]. The present case of ACG2 had typical phenotypic characteristics, including short trunk, small chest, distinctive abdomen and micromyelia, and a radiologic examination revealed short tubular bones with widened metaphyses and non ossified cervical vertebrae, short unfractured ribs, a narrow bellshaped chest, lack of ossification in the pelvis and normal ossification of the skull (Figures 1 and 2).

Apart from skeletal anomalies, severe pulmonary hy-poplasia occurs in ACG2 due to the underlying defects in collagen expression, and respiratory distress can occur immediately after birth with severe forms of the disorder $[2,14]$. The present case had significant respiratory distress immediately after birth, was followed with HFOV due to persistent respiratory distress during mechanical ventilation using conventional settings and the patient died of respiratory insufficiency on postnatal day 25 .

In conclusion, we present a patient with ACG2 and a novel mutation on the $C O L 2 A I$ gene, identified through NGS. Our findings expand the spectrum of causative mutations and clinical findings in ACG2. The authors suggest that the recognition of ACG2 in the prenatal and neonatal period may enable the earlier prediction of the anomaly, and may prolong survival through genetic counseling provided to the family before and after birth.

Declaration of Interest. The authors report no conflicts of interest. The authors alone are responsible for the content and writing of this article.

\section{REFERENCES}

1. Spranger J, Beighton P, Giedion A, Gorlin R, Hall $\mathrm{J}$, Horton B, et al. International Working Group on Constitutional Diseases of Bone. Eur J Pediatr. 1992; 151(6): 407-415.

2. Lee HS, Doh JW, Kim CJ, Chi JG. Achondrogenesis type II (Langer-Saldino achondrogenesis): A case report. J Korean Med Sci. 2000; 15(5): 604-608.

3. Swar MO, Srikrishna BV. Achondrogenesis type II (Langer-Saldino) - A case report. Afr J Med Sci. 1995; 24(3): 297-299.

4. Forzano F, Lituania M, Viassolo A, Superti-Furga V, Wildhardt G, Zabel B, et al. A familial case of achondrogenesis type II caused by a dominant COL2A1 mutation and "patchy" expression in the mosaic father. Am J Med Genet A. 2007; 143A(23): 2815-2820.

5. Robinson JT, Thorvaldsdóttir H, Winckler W, Guttman M, Lander ES, Getz G, et al. Integrative genomics viewer. Nat Biotechnol. 2011; 29(1): 24-26. 
6. Gormez Z, Bakir-Gungor B, Sagiroglu MS. HomSI: A homozygous stretch identifier from next-generation sequencing data. Bioinformatics. 2014; 30(3): 445-447.

7. Zhou X, Chandler N, Deng L, Zhou J, Yuan M, Sun L. Prenatal diagnosis of skeletal dysplasias using a targeted skeletal gene panel. Prenat Diagn. 2018; 38(9): 692-699.

8. Terhal PA, van Dommelen P, Le Merrer M, Zankl A, Simon ME, Smithson SF, et al. Mutation-based growth charts for SEDC and other COL2A1 related dysplasias. Am J Med Genet C Semin Med Genet. 2012; 160C(3): 205-216.

9. Rauch F, Lalic L, Roughley P, Glorieux FH. Genotype-phenotype correlations in nonlethal osteogenesis imperfecta caused by mutations in the helical domain of collagen type I. Eur J Hum Genet. 2010; 18(6): 642-647.

10. Terhal PA, Nievelstein RJ, Verver EJ, Topsakal V, van Dommelen P, Hoornaert K, et al. A study of the clinical and radiological features in a cohort of 93 patients with a COL2A1 mutation causing spondyloepiphyseal dysplasia congenita or a related phenotype. Am J Med Genet A. 2015; 167A(3): 461-475.

11. Barat-Houari M, Sarrabay G, Gatinois V, Fabre A, Dumont B, Genevieve D, et al. Mutation update for COL2A1 gene variants associated with type II collagenopathies. Hum Mutat. 2016; 37(1): 7-15.

12. Kannu $P$, Irving $M$, Aftimos $S$, Savarirayan $R$. Two novel COL2A1 mutations associated with a LeggCalvé-Perthes disease-like presentation. Clin Orthop Relat Res. 2011; 469(6): 1785-1790.

13. Vanegas $\mathbf{S}$, Sua LF, López-Tenorio J, Ramírez-Montaño D, Pachajoa H. Achondrogenesis type IA: Clinical, histologic, molecular, and prenatal ultrasound diagnosis. Appl Clin Genet. 2018; 11: 69-73.

14. Eyre DR, Upton MP, Shapiro FD, Wilkinson RH, Vawter GF. Nonexpression of cartilage type II collagen in a case of Lander-Saldino achondrogenesis. Am J Hum Genet. 1986; 39(1): 52-67. 